\title{
西藏东部地区层析成像及东南部裂谷成因讨论
}

张衡, 赵俊猛, 徐强

中国科学院青藏高原研究所, 大陆碰撞与高原隆升重点实验室, 北京 100085

E-mail: zhangheng415@163.com

2011-03-23 收稿, 2011-05-17 接受

国家重点基础研究发展计划(2008CB425704, 2006CB202301)和国家自然科学基金重点项目(40930317)资助

摘要 利用西藏东部南迦巴瓦地区的 51 个临时地震台站所记录的 169 个远震事件, 从中提取 出 4767 条 $\mathrm{P}$ 波射线, 通过反演计算得到了西藏东部地壳和上地幔的 $\mathrm{P}$ 波速度结构. 水平剖面 显示, 在西藏东南部裂谷附近从近地表到 $250 \mathrm{~km}$ 的深度范围均表现为低速异常; 从纵剖面结 果看, 这个低速异常非垂直地向下延伸到约 $400 \mathrm{~km}$ 深度. 综合分析认为, 此低速异常可能意味 着地幔高温物质上涌, 从而为该区裂谷成因的探讨提供了地震学的证据; 在此低速异常的东侧 出现明显的高速异常, 深度从 $40 \mathrm{~km}$ 延伸到 $200 \mathrm{~km}$, 该高速异常可能是大陆闭合遗留的产物, 并非俯冲的印度板块; 在 250 400 km 的深度范围内, 嘉黎断裂以北为低速异常区, 以南为高速 异常区。该高速异常可能反映了印度板块的北部边界, 并在此发生了拆沉。同时表明, 在西藏 东部印度板块的俯冲未超过嘉黎断裂。

关键词

层析成像

地幔上涌

印度板块俯冲

南北向裂谷

西藏地区
作为世界上平均海拔最高的地区，青藏高原始 终是地球科学研究的热点区域. 自从印度和欧亚板 块在约 $50 \mathrm{Ma}$ 前碰撞以来 ${ }^{[1]}$, 这个地区发生了强烈的 构造变形-地壳缩短、岩石圈增厚、高原隆升、喜马 拉雅山崛起. 如果说雅鲁藏布、班公-怒江、金沙江 等近东西向分布的缝合线是高原形成前特提斯演化 的痕迹, 那么分布于藏南的数个南北向构造则为我 们提出了挑战, 它们是裂谷, 是地堑 ${ }^{[2]}$, 还是深大断 裂? 无疑, 这些近南北走向的构造是青藏高原南部 最显著的构造活动特征, 但到目前它们的属性仍不 清楚, 其形成机制、深浅部构造关系 ${ }^{[3]}$ 仍是认识青藏 高原形成与演化的关键问题.

为研究青藏高原的隆升机制, 在青藏高原上已 开展一系列的国际探测研究计划, 如 INDEPTH 计 划、Hi-CLIMB 计划和 ANTILOPE 计划等, 这些计划 为该区域的深部构造研究提供了许多重要的地震学 证据 ${ }^{[4 \sim 7]}$.

近 20 年来, 随着宽频带地震仪器的大规模使用,
在青藏高原上开展了大量的地震学方面的研究. 面 波探测结果认为, 整个青藏高原下方是相对温度较 低的岩石圈地幔 ${ }^{[8]}$; 走时成像结果认为, 印度板块向 北俯冲穿过了整个青藏高原 ${ }^{[9]}$; 还有学者发现 ${ }^{[10,11]}$, 在西藏中部和北部的上地幔中存在较低的 $\mathrm{S}$ 波速度 异常. Chen 等人 ${ }^{[12]}$ 则认为由于印度板块俯冲到亚洲 板块之下, 从而在西藏大部分地区都产生强烈的径 向各向异性. 利用反射地震学方法, Zhang 等人 ${ }^{[13,14]}$ 提出在 $31.5^{\circ} \mathrm{N}$ 附近的地壳厚度沿东西向有变化, 但 是在 $29^{\circ} \mathrm{N}$ 附近的地壳厚度则无明显变化, 并认为印 度板块在俯冲过程中由高角度俯冲转变为低角度俯冲.

但这些计划和台站大多集中在西藏高原中部和 南部地区, 东部地区的宽频带地震台站很少, 且这些 台站多数是沿垂直于印度板块俯冲方向 (即南北向) 以线性方式架设. 这种线性布台的方式有利于接收 函数方法的研究, 而对走时成像的效果并不理想. 本 文利用南迦巴瓦地区的宽频流动台阵资料, 得到了 西藏东部的地壳与上地幔的速度结构, 为揭示西藏 
东南部裂谷的成因以及印度板块在此地区的俯冲前 缘位置提供了地震学方面的证据.

\section{1 资料与方法}

本研究使用 2003 2004 年位于南迦巴瓦地区的 51 台宽频地震仪(图 1)记录的远震 P 波到时数据. 所 选地震用下列准则进行严格篮选, 以确保结果的准 确性. 具体为: (1) 地震震级大于 5.5 级; (2) 每个 P 波到时需要同时被 5 个以上的台站记录; (3) 震中距 严格控制在 $30^{\circ} \sim 90^{\circ}$ 之间. 研究中全部使用的是 $\mathrm{P}$ 波 初至数据, 拾取精度大约在 $0.1 \sim 0.4 \mathrm{~s}$.

本次研究中所使用的全部地震震源信息来自美 国地质调查局(United States Geological Survey), 它们 的方位如图 2 所示. 可以看出绝大部分地震位于研究 区域的东边, 也就是太平洋板块俯冲区, 这也反映在 相应的射线分布图上(图 3). 研究区域东部的射线覆 盖程度要好于西部, 但整个地区的射线分布效果均 较为理想.

在走时成像的方法中, 将实际到时与理论到时 相减得到绝对走时残差用于反演计算, 这里我们应 用 iasp91 标准模型 ${ }^{[15]}$ 进行相应的理论走时计算. 近 震成像中直接使用绝对走时残差进行计算, 但由于 射线路径都位于模型中, 因而对地震定位有较高的 要求; 在远震中, 则使用相对走时残差(即对每个台 站记录到的绝对走时残差求平均值, 再用每个绝对 残差减去这个平均值)进行计算, 该方法的优点是对 震源位置精度要求不高, 不用进行重新定位. 由于西 藏地区的特殊性一三巨厚的地壳和 $5 \mathrm{~km}$ 的平均海拔, 因此需要将这些影响因素考虑到走时校正中; 同时 在地壳中, 由于远震射线近垂直人射, 使得反演结果

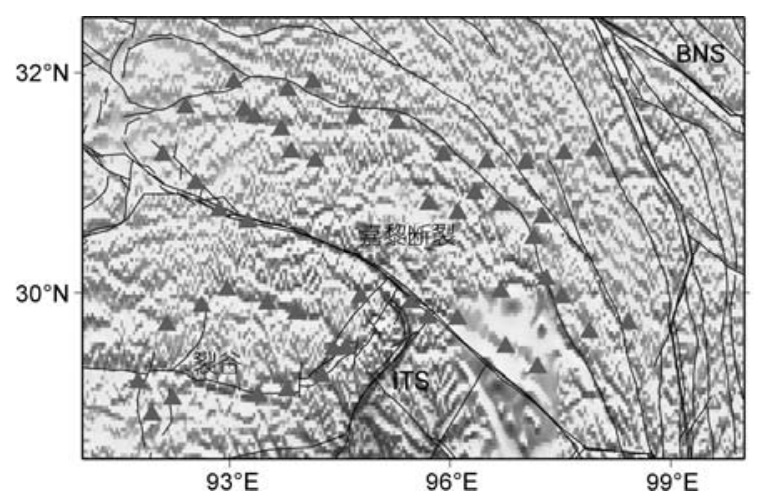

图 1 台站分布及构造示意

三角形代表台站位置; ITS, 雅鲁藏布江㖓合带; BNS, 班公怒江㖓合带

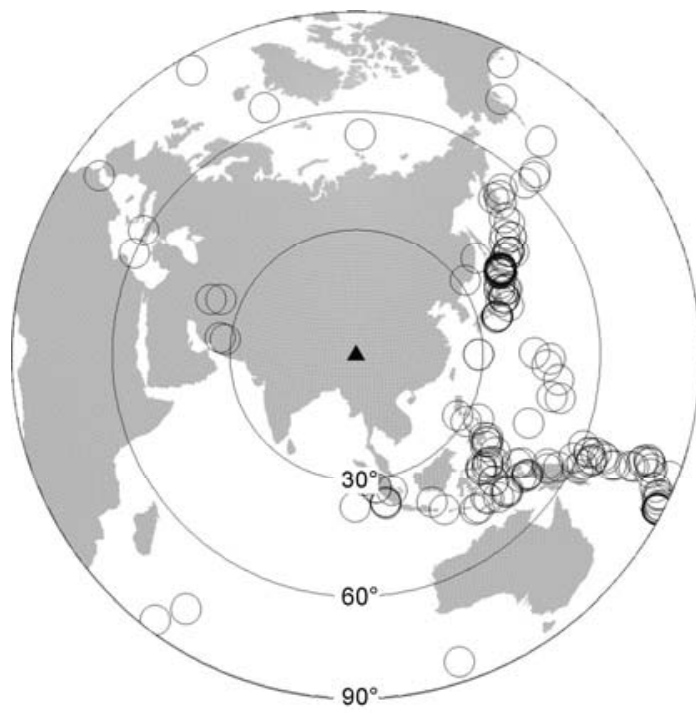

图 2 研究所用地震震中分布

空心圆圈代表地震位置, 实心三角形表示台站中心位置

不是太好, 故而我们使用一种新的校正方法 ${ }^{[16]}$ 来去 除一部分影响.

本次研究采用 Zhao 等人 ${ }^{[17]}$ 的成像方法, 得到了 西藏东南部地区的 $\mathrm{P}$ 波速度结构. 通过多次检测板实 验, 确定了模型的划分在横向上为 $0.5^{\circ}$, 垂向上为 $30 \sim 50 \mathrm{~km}$, 这样的网格划分可以获得最佳的解析精 度. 通过射线追踪, 得到了关于速度扰动的大型稀疏 矩阵, 并采用带阻尼系数和光滑系数 ${ }^{[18]}$ 的 $\mathrm{LSQR}^{[19]}$ 算法求解该矩阵.

\section{2 结果}

检测板实验 ${ }^{[20]}$ 在这里被用来衡量成像结果的质 量(图 4). 从图像上可以看出, 无论是水平剖面(图 4(a)) 还是按照纬度划分的剖面(图 4(b)), 均有较为理想的 分辨还原结果，只是在水平剖面上的 $400 \mathrm{~km}$ 处效果不 太理想, 原因是 $400 \mathrm{~km}$ 是我们设置的模型底界面.

水平剖面的反演结果如图 5 所示. 在地壳深度范 围内 $(40 \sim 70 \mathrm{~km})$, 可以明显看到以 $93^{\circ} \mathrm{E}$ 为界的高低 速异常分布, 低速异常在地表直接对应着该地区裂 谷的位置, 且与这些裂谷的走向基本一致. 在 100 $\mathrm{km}$ 剖面图上这条高低速异常分界线依然明显, 只是 高低速异常的幅度有所减小. 从 150 200 km 剖面上, 近东西向的高低速异常分布特征几乎消失, 转而为 近南北向分布, 嘉黎断裂以南主要为低速异常, 以北 则主要为高速异常. 从 $250 \mathrm{~km}$ 直到 $400 \mathrm{~km}$ 深度剖面 

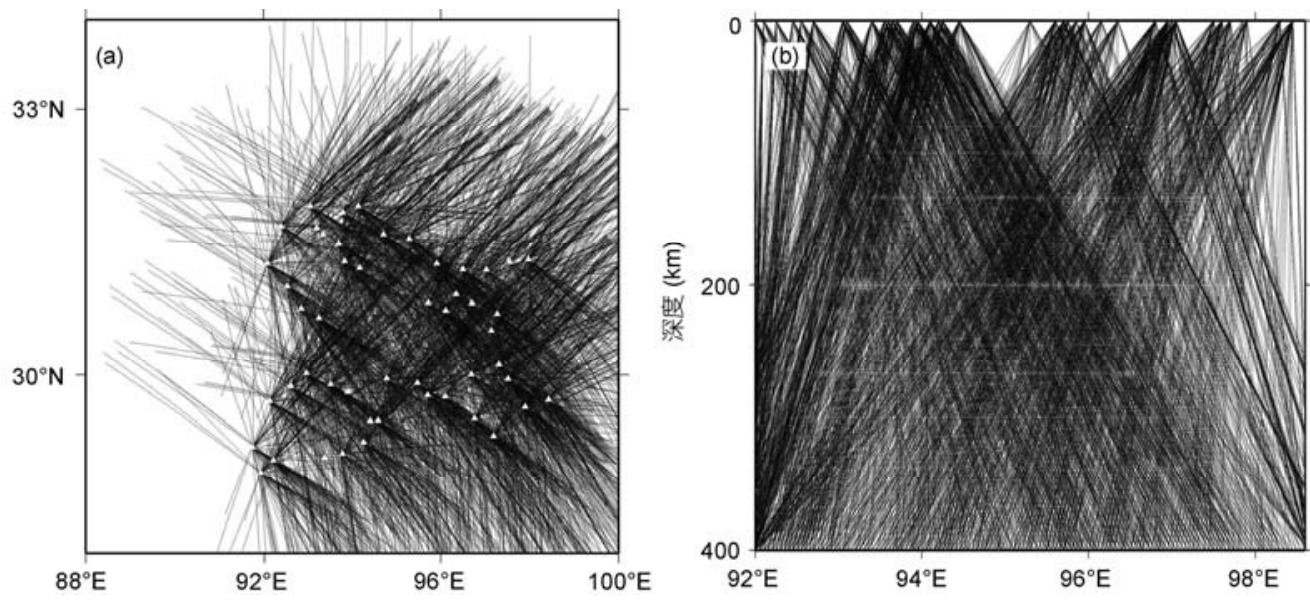

图 3 研究区域射线分布

(a) 水平剖面; (b) 垂向剖面

图 4 水平 $(a)$ 和纬度(b)剖面检测板实验

(尽管 $400 \mathrm{~km}$ 剖面位于模型边界处, 但主要地区的分 辨率依然较好), 这种以嘉黎断裂为分界线的近南 北向分布的高低速异常特征越加明显, 只是嘉黎 断裂以南主要呈现出高速异常, 而以北则是低速 异常为主.

以分辨率最好的 $30^{\circ} \mathrm{N}$ 剖面(图 4(b)) 为例，观察 西藏东部地下结构的东西向变化(图 6). 该图像从西 至东依次呈现出高-低-高速异常, $92^{\circ} \mathrm{E}$ 附近的高速异 常只存在于 200 400 km 之间; 位于图中部的低速异 常从地表一直延伸到 $400 \mathrm{~km}$, 且在约 $200 \mathrm{~km}$ 处开始 向东倾斜; 在这条东倾的低速带之上 $(0$ 200 km), 呈 现出明显的高速异常.

\section{3 分析}

图 5 的浅层 $(40 \sim 70 \mathrm{~km})$ 速度异常分布与该地区的 很多地球物理研究结果类似, 比如 Pn 波成像结果显

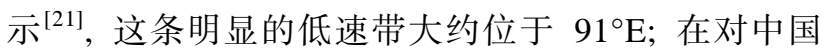
及周边地区的大范围成像结果中 ${ }^{[22]}$, 高原东部的这 条低速异常带的位置与我们的结果几乎一致. 上述 两项结论均未使用南迦巴瓦台站的数据, 但是在相 近的位置同样发现了这样一条低速异常带. 在使用 了此地区台站数据后, 全球体波成像结果显示 ${ }^{[23]}$, 在 $90^{\circ} \mathrm{E}$ 附近, 雅江以北的位置存在一条由地表至 $310 \mathrm{~km}$ 的低速异常带; 而面波研究 ${ }^{[24,25]}$ 也得出了相 似结果. 除了这条明显的低速异常带, 在该异常带的 


\section{图 5 成像结果水平剖面}

东边 $(0 \sim 200 \mathrm{~km})$ 呈现出明显的高速异常. 在相同地区 的走时成像结果中 ${ }^{[26]}$, 印度板块俯冲的前沿位置仅 到达 $30^{\circ} \mathrm{N}$ 附近, 且越靠近东边俯冲的距离越短. 利 用相同的地震台站数据, Ren 等人 ${ }^{[27]}$ 使用有限频成像 技术也发现了这样的高速异常, 并提出与 Pn 波成像 结果 ${ }^{[21]}$ 类似的解释, 认为在西藏东部地区, 靠近东 侧的印度板块俯冲距离更长. 在地壳深度范围内, 与 同区或相近区域的地球物理结果相比, 此高速异常 均不明显, 甚至是存在明显低速异常 (这可能与方法 和数据本身的局限有关). 比如, Makovsky 等人 ${ }^{[28]}$ 通 过对人工源地震数据资料进行处理, 认为亚东-谷露 裂谷附近的中下地壳之中存在自由水; 使用 Rayleigh
波成像技术和该地区地震资料, Fu 等人 ${ }^{[29]}$ 在较浅深 度(Moho 面深度之上)并未发现此高速异常，该结论 与 $\mathrm{Bai}$ 等人 ${ }^{[30]}$ 和赵国泽等人 ${ }^{[31]}$ 的大地电磁成果较为 吻合, 同时也与 Zhang 等人 ${ }^{[13]}$ 通过反射地震方法得 到的结果比较一致(嘉黎断裂附近出现部分熔融); 在 该地区同样利用 Rayleigh 波和 Love 波成像技术(但使 用不同的数据), Chen 等人 ${ }^{[32]}$ 也发现了极低速异常 (7\% 10\%), 从而认为此处满足中下地壳流动的条件. 而在 70 200 km 深度, 本文图像与 $\mathrm{Fu}$ 等人 ${ }^{[29]}$ 都发现 了明显高速异常. 关于此高速异常, Kumar 等人 ${ }^{[33]}$ 以 及 $\mathrm{Fu}$ 等人 ${ }^{[29]}$ 等人均认为是亚洲岩石圈的俯冲造成. 对比于一些该地区的其他成像结果, 如 $\mathrm{Li}$ 等人 ${ }^{[26]}$ 在 
Burma 和腾冲附近的成像没有在 $98^{\circ} \mathrm{E}$ 以西发现到这 样的高速异常; 藏东南地区的龙门断裂附近的走时 成像结果 ${ }^{[34,35]}$ 也并无证据表明亚洲岩石圈直接俯冲 到了青藏高原东部。综上所述, 并且同以往的结果相 比 ${ }^{[21,22,36]}$, 在这条低速带以西并未发现明显的高速异 常, 故而认为这种高速异常并非由现在的印度板块 俯冲造成, 而是由于地幔物质上涌, 带动已经下沉的 大陆闭合时的物质随之上升产生的. 从 $200 \mathrm{~km}$ 开始, 嘉黎断裂以南表现出的明显高速异常(图 5), 以北则 表现出明显的低速异常. 由此认为, 印度板块的俯冲 没有越过嘉黎断裂，而是在喜马拉雅地体下方产 生了拆沉. 另外, 虽然在 $100 \mathrm{~km}$ 以上不能以明显 的速度异常分界线确定嘉黎断裂，但是从 $150 \mathrm{~km}$ 处开始, 以嘉黎断裂为界, 速度结构呈现出明显的 南北向趋势. 关于产生这种现象的原因, 还需要进一 步的研究.

利用接收函数方法, Singh 等人 ${ }^{[37]}$ 发现了该地区 的明显低速异常是由于拆沉至 $410 \mathrm{~km}$ 间断面之下的 高速物质(印度岩石圈地幔)所引起的. 尽管我们认为 造成该地区裂谷的主要原因是地幔物质上涌, 但是 从图 6 来看, 这种上涌的趋势并非是简单的垂向上涌, 而是呈现出一种向东倾斜的态势. 这种倾斜的上涌 模式在冰岛、夏威夷、贝加尔等地都被发现过 ${ }^{[36]}$, 虽 然这个地区的裂谷范围不如贝加尔裂谷明显，相关 的研究也很少, 但是从检测板实验(图 4(b))的结果来 看, 这个剖面的还原度非常好; 除此之外, 通过计算 机模拟, 得出地幔的流动会使地幔柱产生倾斜 ${ }^{[38]}$, 而青藏高原的地幔物质具有向东流动的趋势 ${ }^{[4]}$; 并且 通过 $\mathrm{P}$ 波速度与 $\mathrm{S}$ 波速度比值的比较, 其结果指出这 里的低速物质并非是简单的由于地幔的温度升高而 引起 ${ }^{[27]}$. 综上所述, 认为这种倾斜的地幔上涌是存 在的.

在这条低速带的西边 $\left(94^{\circ} \mathrm{E}\right.$ 以西), 有一处由 100 $\mathrm{km}$ 深度延伸至 $400 \mathrm{~km}$ 的高速异常体. 虽然靠近成像 边界处会有一定的边界效应，但是总体位置 $\left(92^{\circ} \sim 94^{\circ} \mathrm{E}\right)$ 的还原度还是可信的. 从该高速体的位置, 以及与 INDEPTH 计划的一些结果相比较 ${ }^{[5]}$, 将这个 高速块体解释为俯冲的印度板块, 并认为可能是由

\section{图 $630^{\circ} \mathrm{N}$ 剖面}

于俯冲而导致了临近区域的地幔柱上涌.

本次研究中, 由于流动地震台站的位置限制, 仅 能得出西藏东南部裂谷的地下结构，而该地区的裂 谷成因能否推广到整个西藏南部的南北向裂谷, 还 有待于进一步的研究. 为了能更深人地研究这些裂 谷的成因和地下结构, 尚需布设更多的地震流动台站.

\section{4 结论}

本次研究使用了西藏东部南迦巴瓦地区流动地 震台站的数据, 通过远震层析成像方法得到了该地 区地壳与上地幔的速度结构. 在深度 $100 \mathrm{~km}$ 以上, 该地区的速度结构呈现出明显的东西向分布特征; 在 150 400 km 的范围, 这种速度异常的分布由近东 西向转变为近南北向, 且以嘉黎断裂为界. 研究认为, 印度板块俯冲至喜马拉雅地体下发生了拆沉, 深度 大约在 200 350 km 之间, 俯冲前缘并未超过嘉黎断 裂. 出现在 $92^{\circ} \mathrm{E}$ 附近的低速异常(其强弱达到 $2 \%$ )是 地幔物质的倾斜上涌造成的，这也是地表裂谷的主 要原因; 而产生这种倾斜上涌则是由于地幔物质的 横向流动造成的.

致谢文中所使用原始数据来自南迦巴瓦地区临时地震台站, 并通过 IRIS 数据中心下载. 感谢日本东北大学赵大鹏教 授提供的 Tomog $3 \mathrm{~d}$ 程序. 


\section{参考文献}

1 Yin A, Harrison T M. Geologic evolution of the Himalayan-Tibetan orogen. Annu Rev Earth Planet Sci, 2000, 28: 211-280

2 吴中海, 张永双, 胡道功, 等. 西藏错那-拿日雍错地堑的第四纪正断层作用及其形成机制探讨. 第四纪研究, 2008, 28: 232-242

3 Jimenez-Munt I, Platt J P. Influence of mantle dynamics on the topographic evolution of the Tibetan Plateau: Results from numerical modeling. Tectonics, 2006, 25, doi:10.1029/2006TC001963

4 Zhao J M, Yuan X H, Liu H B, et al. The boundary between the Indian and Asian tectonic plates below Tibet. Proc Natl Acad Sci USA, 2010, 107: 11229-11233

5 Tilmann F, Ni J, Team I I S. Seismic imaging of the downwelling Indian lithosphere beneath central Tibet. Science, 2003, 300: 1424-1427

6 Nabelek J, Hetenyi G, Vergne J, et al. Underplating in the Himalaya-Tibet Collision Zone Revealed by the Hi-CLIMB Experiment. Science, 2009, 325: 1371-1374

7 苏伟, 王椿镛, 黄忠贤. 青藏高原及邻区的 Rayleigh 面波的方位各向异性. 中国科学 D 辑：地球科学, 2008, 38: 674-682

8 Priestley K, Debayle E, McKenzie D, et al. Upper mantle structure of eastern Asia from multimode surface waveform tomography. $\mathrm{J}$ Geophys Res, 2006, 111, doi:10.1029/2005JB004082

9 Zhou H W, Murphy M A. Tornographic evidence for wholesale underthrusting of India beneath the entire Tibetan plateau. J Asian Earth Sci, 2005, 25: 445-457

10 Rapine R, Tilmann F, West M, et al. Crustal structure of northern and southern Tibet from surface wave dispersion analysis. J Geophys Res, 2003, 108, doi:10.1029/2001JB000445

11 Lebedev S, van der Hilst R D. Global upper-mantle tomography with the automated multimode inversion of surface and S-wave forms. Geophys J Int, 2008, 173: 505-518

12 Chen Y, Badal J, Zhang Z J. Radial anisotropy in the crust and upper mantle beneath the Qinghai-Tibet Plateau and surrounding regions. J Asian Earth Sci, 2009, 36: 289-302

13 Zhang Z J, Klemperer S. Crustal structure of the Tethyan Himalaya, southern Tibet: New constraints from old wide-angle seismic data. Geophys J Int, 2010, 181: 1247-1260

14 Zhang Z J, Klemperer S L. West-east variation in crustal thickness in northern Lhasa block, central Tibet, from deep seismic sounding data. J Geophys Res, 2005, 110, doi:10.1029/2004JB003139

15 Kennett B L N, Engdahl E R. Traveltimes for global earthquake location and phase identification. Geophys J Int, 1991, 105: 429-465

16 江国明, 赵大鹏, 张贵宾. 远震层析成像中的地壳校正研究及应用. 地球物理学报, 2009, 52: 1508-1514

17 Zhao D P, Hasegawa A, Horiuchi S. Deep structure of Japan subduction zone as derived from local, regional, and teleseismic events. J Geophys Res, 1994, 99: 22313-22329

18 Paige C C, Saunders M A. LSQR: An Algorithm for Sparse Linear-Equations and Sparse Least-Squares. ACM Trans Math Softw, 1982, 8: $43-71$

19 Zhao D. Seismic structure and origin of hotspots and mantle plumes. Earth Planet Sci Lett, 2001, 192: 251-265

20 Humphreys E, Clayton R W. Adaptation of back projection tomography to seismic travel time problems. J Geophys Res, 1988, 93: $1073-1085$

21 Liang C T, Song X D. A low velocity belt beneath northern and eastern Tibetan Plateau from Pn tomography. Geophys Res Lett, 2006, 33, doi:10.1029/2006GL027926

22 Huang J L, Zhao D P. High-resolution mantle tomography of China and surrounding regions. J Geophys Res, 2006, 111, doi:10.1029/2005JB004066

23 周华伟, Murphy M A, 林清良. 西藏及其周围地区地壳、地幔地震层析成像一印度板块大规模俯冲于西藏高原之下的证据. 地学 前缘, 2002, 9: 285-292

24 孙若昧, 张中杰, 滕吉文. 青藏高原物质侧向流动的讨论一面波层析成像的证据. 见: 张中杰, 高锐, 吕庆田, 等, 编. 中国大陆 地球深部结构与动力学研究. 北京: 科学出版社, 2004. 872-880

25 张雪梅, 孙若昧, 滕吉文. 青藏高原及其邻区地壳、岩石圈和软流层厚度研究. 科学通报, 2007, 52: 332-338

26 Li C, Van der Hilst R D, Meltzer A S, et al. Subduction of the Indian lithosphere beneath the Tibetan Plateau and Burma. Earth Planet Sci Lett, 2008, 274: 157-168

27 Ren Y, Shen Y. Finite frequency tomography in southeastern Tibet: Evidence for the causal relationship between mantle lithosphere delamination and the north-south trending rats. J Geophys Res, 2008, 113, doi:10.1029/2008JB005615

28 Makovsky Y, Klemperer S L. Measuring the seismic properties of Tibetan bright spats: Evidence for free aqueous fluids in the Tibetan middle crust. J Geophys Res, 1999, 104: 10795-10825 
29 Fu Y Y V, Li A B, Chen Y J. Crustal and upper mantle structure of southeast Tibet from Rayleigh wave tomography. J Geophys Res, 2010, 115, doi:10.1029/2009JB007160

30 Bai D H, Unsworth M J, Meju M A, et al. Crustal deformation of the eastern Tibetan plateau revealed by magnetotelluric imaging. Nat Geosci, 2010, 3: 358-362

31 赵国泽，陈小斌，王立凤，等. 青藏高原东边缘地壳“管流”层的电磁探测证据. 科学通报, 2008, 53: 345-350

32 Chen Y, Badal J, Hu J F. Love and Rayleigh wave tomography of the Qinghai-Tibet Plateau and surrounding areas. Pure Appl Geophys, 2010, 167: 1171-1203

33 Kumar P, Yuan X H, Kind R, et al. Imaging the colliding Indian and Asian lithospheric plates beneath Tibet. J Geophys Res, 2006, 111, doi:10.1029/2008JB005615

34 韦伟, 孙若昧, 石耀霖. 青藏高原东南缘地震层析成像及汶川地震成因探讨. 中国科学：地球科学, 2010, 40: 831-839

35 李志伟, 胥䝠, 黄润秋. 龙门山地区的 P 波速度结构与汶川地震的深部构造特征. 中国科学：地球科学, 2011, 41: 283-290

36 Zhao D P. Global tomographic images of mantle plumes and subducting slabs: Insight into deep Earth dynamics. Phys Earth Planet Inter, 2004, 146: 3-34

37 Singh A, Kumar M R. Seismic signatures of detached lithospheric fragments in the mantle beneath eastern Himalaya and southern Tibet. Earth Planet Sci Lett, 2009, 288: 279-290

38 Steinberger B. Plumes in a convecting mantle: Models and observations for individual hotspots. J Geophys Res, 2000, 105: 11127-11152 